\title{
3D trajectories and diffusion of single ceria particles near a glass surface and their removal
}

\author{
Jihoon Seo ${ }^{1,2, a), c)}\left(\mathbb{D}\right.$, Akshay Gowda ${ }^{1, c}$, Panart Khajornrungruang ${ }^{3}$ (i), Satomi Hamada ${ }^{4}$, \\ S.V. Babul ${ }^{1,2, b)}$ \\ ${ }^{1}$ Department of Chemical and Biomolecular Engineering, Clarkson University, Potsdam, New York 13699, USA \\ ${ }^{2}$ Center for Advanced Materials Processing, Clarkson University, Potsdam, New York 13699, USA \\ ${ }^{3}$ Department of Mechanical Information Science and Technology, Kyushu Institute of Technology, Iizuka-shi, Fukuoka-ken 820-8502, Japan \\ ${ }^{4}$ Advanced Technology Division, EBARA Corporation, Fujisawa-shi, Kanagawa 251-8502, Japan \\ Address all correspondence to these authors. \\ a)e-mail: jseo@clarkson.edu \\ b)e-mail: babu@clarkson.edu \\ ${ }^{c}$ Authors J.S. and A.G. contributed equally to this work.
}

Received: 5 May 2020; accepted: 24 August 2020; published online: 2 February 2021

We extend our recent 2D trajectory ( $x-y$ plane) and diffusion coefficient data of ceria particles near a glass surface obtained at $\mathrm{pH} 3,5$, and 7 using evanescent wave microscopy and video imaging to 3D trajectories by analyzing the separation distance between the particles and the glass surface in the vertical $z$-direction. Mean squared displacement $\left(\mathrm{MSD}_{3 \mathrm{D}}\right.$ ) of ceria particles was calculated to quantify 3D trajectories. Three-dimensional diffusion coefficients were obtained from the $\mathrm{MSD}_{3 \mathrm{D}}$ curves and were compared with two-dimensional diffusion coefficients. By analyzing the MSD curves, we found that ceria particles exhibited only confined motion at pH 3 and 5, while both confined and Brownian motion were showed at $\mathrm{pH}$ 7. We also evaluated the cleaning ability of DI water adjusted to $\mathrm{pH} 10$ and 12 to remove ceria particles from glass surfaces and related the results to the calculated trajectory, diffusion coefficient, and interaction potential data.

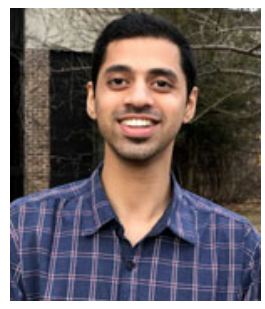

\footnotetext{
Dr. Akshay Gowda is currently working as a Chemical Mechanical Planarization (CMP) Process Development Engineer at Intel Corporation, Rio Rancho, NM, USA. He works on 3D XPoint technology for data storage applications by collaborating with different teams and tool vendors to accomplish program goals. His primary job is to plan and execute experiments to meet process requirements utilizing statistical knowledge and best problem-solving techniques. He received his Bachelor of Technology degree in Chemical Engineering from National Institute of Technology Karnataka, India in 2016, and his Ph.D. degree in Chemical and Biomolecular Engineering from Clarkson University, Postdam, NY, USA in 2020. During his Ph.D. at Clarkson University, he worked with Dr. S.V. Babu, Distinguished University Professor in Department of Chemical and Biomolecular Engineering. His Ph.D. thesis focused on developing cleaning chemistries for removal of ceria particles from silicon dioxide surfaces post-CMP of shallow trench isolation (STI) structures. A major part of his research at Clarkson University concentrated on understanding ceria particle-silicon dioxide surface interactions from a fundamental perspective using evanescent wave (EW) microscopy. The interactions of ceria particles with glass film (representative of silicon dioxide surface) were studied in real time using homebuilt EW microscopy and cleaning strategies to remove ceria particles from silicon dioxide wafers by imaging them using atomic force microscopy (AFM) were developed later. He also worked on developing CMP slurries for polishing amorphous silicon carbonitride, silicon nitride, silicon dioxide and Cobalt films for different applications by collaborating with several different industries during his Ph.D. He interned at Cabot Microelectronics in the summer of 2019 where he worked on developing a model to predict CMP polishing pad and conditioner lifetime.
} 


\section{Introduction}

A detailed understanding of the interaction of particles with various films has received significant interest over the past few decades [1, 2, 3, 4]. In particular, in chemical mechanical planarization (CMP) applications, the interaction of ceria particles with $\mathrm{SiO}_{2}$ films has been extensively studied to understand the influence of slurry chemistry, interaction force and particle adsorption on $\mathrm{SiO}_{2}$ films and particle removal during polishing and cleaning, respectively $[5,6,7,8]$. Many studies have demonstrated that atomic force microscopy (AFM) and the quartz crystal microbalance-dissipation (QCM-D) can be used to investigate the particle-surface interaction forces and the adsorbed amount, respectively $[8,9,10,11]$. AFM colloidal probe technique has been widely used to measure directly the interactions between ceria particles and surfaces. However, the interaction forces can be influenced by the external forces applied to keep the particles attached (glued) to the AFM cantilever. Similarly, while QCM-D is useful for monitoring the real-time adsorption/desorption behavior of particles on surfaces (i.e., adsorption/desorption rates and their amounts), it provides limited information on the movements of particles on the surface and their interaction energies.

We recently reported that evanescent wave (EW) microscopy, also known as total internal reflection microscopy (TIRM), is a powerful tool to directly measure interactions between ceria particles $\left(d_{\text {mean }} \sim 140 \mathrm{~nm}\right)$ and glass surfaces [12]. Our home-built EW microscopy setup and a schematic of the acquired data and analysis are shown in Fig. 1. The EW was generated by the total internal reflection (TIR) of green laser light at the ceria suspension/glass interface. In all our experiments, the flat glass surface of the hemispherical lens shown in Fig. 1 became our model for the $\mathrm{SiO}_{2}$ film surface. The details of the EW microscopy system and the measurement conditions were described earlier [12] and and are also summarized later in the Experimental section of this paper.

Earlier, the in situ trajectories, diffusion, and interaction energies of several single ceria particles and, in some cases, their agglomerates near a glass surface in $2 \mathrm{D}(x-y)$ plane were obtained without the application of any external forces [12]. Although this provides useful information on the particle dynamics near a glass surface [12], the real space is three dimensional. Thus, a 3D trajectory analysis can provide more reliable information on the interactions of ceria particles with a glass surface, including adsorption and their removal near a glass surface. In this paper, by combining the data on the distance of the particles from the glass surface in the $z$-direction (height, $h$ ) with $2 \mathrm{D}$ data, we obtain $3 \mathrm{D}$ trajectories

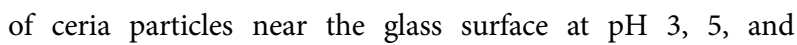
7. Three-dimensional diffusion coefficients $\left(D_{3 \mathrm{D}}\right)$ were then obtained from the calculated mean square displacement

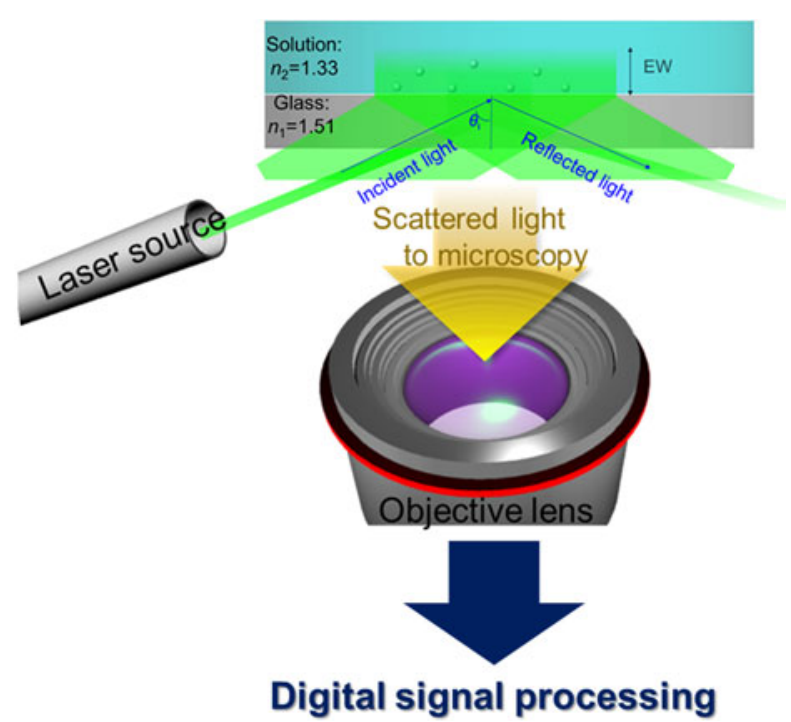

Figure 1: Schematic of our home-built EW microscopy setup (modified from Ref. [12] with permission from the Journal of Materials Research). Processing of incoming data in real time with digital signal processing was reported previously $[12,13,14]$; see the Experimental section for details.

$\left(\mathrm{MSD}_{3 \mathrm{D}}\right)$ (see the details in the section "3D trajectory analysis") and were compared with $D_{2 \mathrm{D}}$ values obtained in our previous paper [12]. 3D trajectories and $D_{3 \mathrm{D}}$ values of the ceria particles depend on the type and characteristics of particle motion and are influenced by $\mathrm{pH}$ values.

While many studies have exploited EW microscopy to study particle interactions with various surfaces, very few studies have used EW microscopy to investigate the removal of ceria particles from surfaces $[15,16]$. Here, we show that this technique also enables the monitoring of the removal of ceria particles from a glass surface in real time. The resulting data are of interest for the post-CMP cleaning of ceria particles from $\mathrm{SiO}_{2}$ film surfaces.

\section{Results and Discussion}

\section{D trajectory analysis of a single ceria particle with confined motion near a glass surface}

We demonstrate here an expeditious method for obtaining 3D trajectories of single ceria particles at three different $\mathrm{pH}$ values using data obtained from EW microscopy coupled with video imaging (Fig. 2). Positions (i.e., centroids) of single ceria particles near a glass surface in each frame were identified from local maxima in their EW scattering intensity fitted to a Gaussian function using the particle tracking software [17]. 2D trajectories of a single ceria particle were obtained by linking its timesequenced positions (upper figure in Fig. 2). The distance of the particle from the glass surface in the $z$-direction (height, h) can be obtained using the Beer-Lambert relation between 

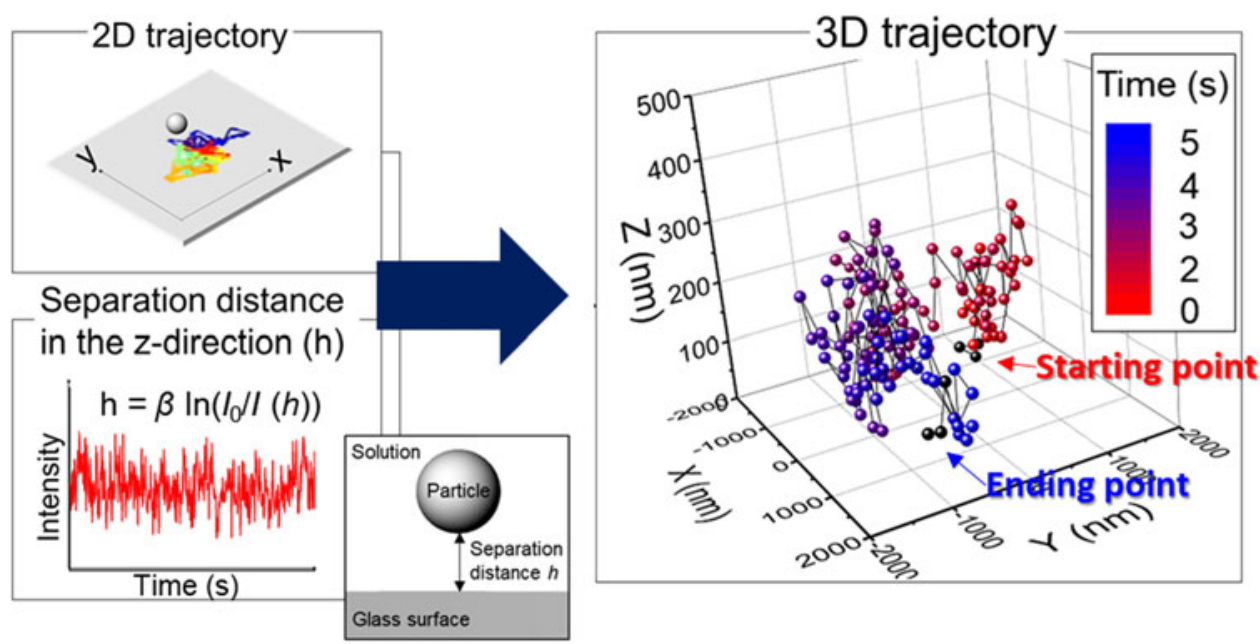

Figure 2: Trajectory of a single ceria particle near a glass surface in $2 \mathrm{D}(x-y)$ plane (upper figure where each colored track shows the particle's movement between two different frames). EW intensity of a single particle versus time data is shown in the lower figure. The 3D trajectory of a single ceria particle was obtained by combining the $2 \mathrm{D}(x-y)$ data with the separation distance $h$ (see the next section for details). The first and last three points of the 3D trajectory were marked as black dots to determine the beginning and the end of the particle movement.

$h$ and the EW intensity (lower figure in Fig. 2), $h(I)=\beta \ln \left(I_{0} / I\right)$ $[18,19]$, where $\beta(88.0 \pm 4.5 \mathrm{~nm})$ is the penetration depth of the laser beam, $I$ is the EW scattering intensity of the ceria particle, and $I_{0}$ is the maximum intensity obtained at the particle/surface interface [18]. EW intensity is maximum at the glass surface and decays exponentially with the distance $h$ according to $I$ $(h)=I_{0} \exp \left(-\beta^{-1} h\right)[18,19]$. The penetration depth $\beta$ is defined as the distance from the glass surface where EW intensity decays to $1 / e$ or $\sim 37 \%$ of $I_{0}[18,19]$. It should be noted that the imaging depth is not limited to the penetration depth and particles located beyond the EW penetration depth from the glass surface can also be imaged [19,20,21,22,23]. For example, Prieve and Walz reported smaller than $400 \mathrm{~nm}$ separation distance using a $7 \mu \mathrm{m}$ polystyrene sphere with a penetration depth of $197 \mathrm{~nm}$ [19]. McKee et al. calibrated the relationship between EW intensity and particle-surface distance and showed that the effective range of separation distance using a $1.2 \mu \mathrm{m}$ glass particle and a penetration depth of $66.8 \mathrm{~nm}$ was about three times the penetration depth [21]. The separation distances $h$ of ceria particles near a glass surface, obtained from the intensity data in our paper, are less than $\sim 3$ times the penetration depth and thus can be used as the distance $h$ for their 3D positions. 3D positions of the particles were determined by combining $2 \mathrm{D}$ positions with the associated separation distance $h$ in each frame, and then linking them to generate 3D trajectories (Fig. 2).

$50 \mu \mathrm{L}$ of a dilute ceria suspension was placed on the flat surface of the glass lens and imaged in situ using EW microscopy for $20 \mathrm{~s}$. The number of ceria particles on the glass surface, obtained from EW microscopy images and videos, is $\sim 2950$ at $\mathrm{pH} 3, \sim 1530$ at $\mathrm{pH} \mathrm{5}$, and $\sim 427$ at $\mathrm{pH} 7$ in the imaged

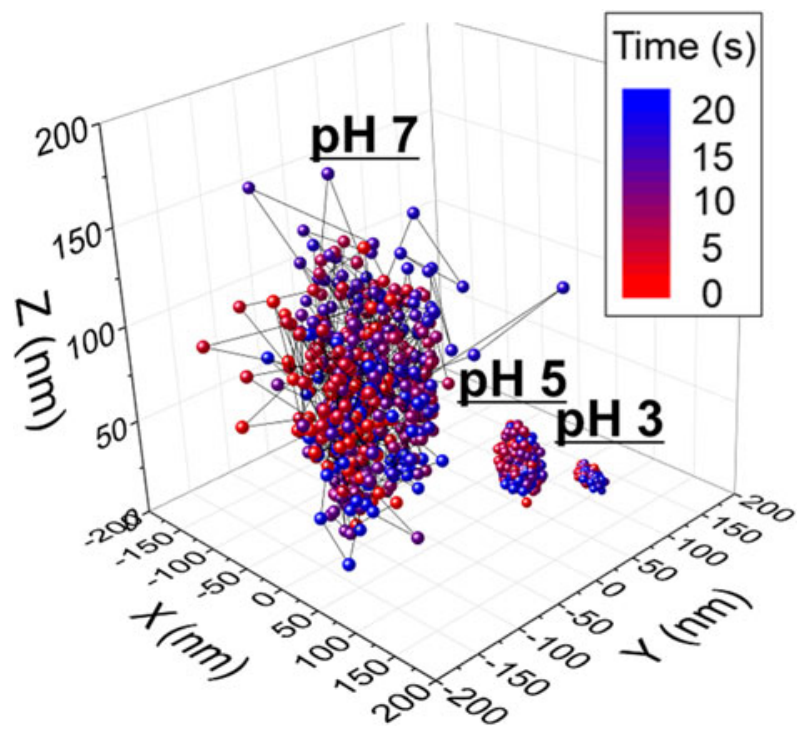

Figure 3: $3 \mathrm{D}$ trajectories of three different single ceria particles near a glass surface at $\mathrm{pH} 3,5$, and 7 . These data were obtained from individual experiments performed at three different $\mathrm{pH}$ values and combined into a single figure.

area of $77 \times 77 \mu \mathrm{m}^{2}$, corresponding to $\sim 0.5, \sim 0.3$, and $\sim 0.1$ particles $/ \mu \mathrm{m}^{2}$, respectively. Thus, the concentrations of the ceria particles on the glass surface are sufficiently low, so that particle-particle interactions are negligible. Indeed, to minimize the effect of particle-particle interactions in our analysis, only particles that were more than 10 particle diameters apart from neighboring particles were monitored. For trajectory analysis, three different single ceria particles each at three different $\mathrm{pH}$ values were monitored as representative samples. Figure 3 shows representative 3D and 2D trajectories 
of one of these three ceria particles, tracked for 500 frames over a total time of $20 \mathrm{~s}$, at $\mathrm{pH} \mathrm{3,5}$, and 7. These data were obtained from separate experiments performed at each $\mathrm{pH}$ value and combined into a single figure. In Fig. 3, the time evolution of these positions can be visualized from the color scheme that changes gradually from red initially $(t=0)$ to blue at $t=20 \mathrm{~s}$.

The movements of single ceria particles at $\mathrm{pH} 3$ and 5 are confined to a relatively small area of $15 \times 15 \mathrm{~nm}^{2}$ and $30 \times$ $30 \mathrm{~nm}^{2}$, respectively, due to strong attractive electrostatic and van der Waals forces (zeta-potentials of ceria particle at $\mathrm{pH} 3$ and 5 are 70 and $50 \mathrm{mV}$, respectively, and that of $\mathrm{SiO}_{2}$ film is $-20.0 \mathrm{mV})$ [12]. The isoelectric point $\left(\mathrm{pH}_{\mathrm{IEP}}\right)$ of the glass $\left(\mathrm{SiO}_{2}\right)$ film is 2.0 , resulting in a negatively charged glass surface in the $\mathrm{pH}$ range we are interested in. The vertical separation distance $h$, obtained from the intensity data, showed that the particles were also confined in the $z$-direction over a height of about 15 and $45 \mathrm{~nm}$ at $\mathrm{pH} 3$ and 5, respectively. By contrast, the movements of single ceria particles near the glass surface at $\mathrm{pH} 7$, though still confined, covered an area of $300 \times 300 \mathrm{~nm}^{2}$ and a vertical distance along a $z$-axis of $150 \mathrm{~nm}$, presumably due to the consequence of the weaker attractive forces between the ceria particle and the glass surface (zeta-potential of ceria particle at $\mathrm{pH} 7$ is close to $0 \mathrm{mV}$ and that of $\mathrm{SiO}_{2}$ film is $-34.0 \mathrm{mV}$ ) [12]. Different types and characteristics of particle motions observed at $\mathrm{pH} 7$ will be discussed in the subsequent section. The increases seen in the space covered by the particles with $\mathrm{pH}$ corroborate the findings from potential energy curves that showed that the separation distance increases with $\mathrm{pH}$ due to weakening of the attractive forces at higher $\mathrm{pH}$ values [12].

Mean square displacement (MSD) of any particle along its trajectory can be obtained at various lag times $\tau_{n}\left(\tau_{n}=n \Delta t\right.$, $n=1,2, \ldots N-1)$ using the definition [24,25]

$\mathrm{MSD}_{3 \mathrm{D}}=\frac{1}{N-n} \sum_{i=0}^{N-n}\left(\left(x_{i+n}-x_{i}\right)^{2}+\left(y_{i+n}-y_{i}\right)^{2}+\left(z_{i+n}-z_{i}\right)^{2}\right)$,

where $N$ is the total number of time frames $(N=500)$ over which the trajectories were imaged, $\Delta t$ is the time interval between two consecutive image frames ( $40 \mathrm{~ms}$ in our case) defined by the frame acquisition rate, and $n$ is the number of the time intervals. Also, $\left(x_{i+n}, y_{i+n}\right.$, and $\left.z_{i+n}\right)$ are the positions of the particle at time $n \Delta t$ after starting at position $\left(x_{i}, y_{i}\right.$, and $z_{i}$ ) at $t=0$. Again, at any time, the particle positions are given by those of its centroid where the highest intensity occurs, determined by fitting a Gaussian profile using the Diatrack software as described in the Experimental section.

Figure 4 shows the $\mathrm{MSD}_{3 \mathrm{D}}$ curves at three different $\mathrm{pH}$ values. $\mathrm{MSD}_{3 \mathrm{D}}$ of single ceria particles at $\mathrm{pH} \mathrm{3,5}$, and 7 increased with increasing $\tau$, and this trend is similar to that observed in the case of $2 \mathrm{D}$ data [12]. If a particle shows anomalous diffusion, its $\mathrm{MSD}_{3 \mathrm{D}}$ exhibits a nonlinear relationship with $\tau$, expressed by a power law of the form $\mathrm{MSD}_{3 \mathrm{D}}=6 D_{3 \mathrm{D}} \tau^{a}$ where $D_{3 \mathrm{D}}$ is three-dimensional diffusion coefficient and $a$ is the exponent that determines the types and characteristics of particle motions [26,27,28]. If $0<a<1$, the motion is said to be confined or sub-diffusive, Brownian if $a=1$, and superdiffusive if $a>1 \quad[26,27,28]$. In our case, the value of the exponent $a$ obtained by fitting the data is $\sim 0.11$ for $\mathrm{pH} 3$ and 5 and $\sim 0.14$ for $\mathrm{pH} 7$ confirming confined motion of the particle. The associated three-dimensional diffusion coefficients $D_{3 \mathrm{D}}$ are $1.4 \times 10^{-5}, 6.7 \times 10^{-5}$, and $1.0 \times 10^{-3} \mu \mathrm{m}^{2} / \mathrm{s}$, at $\mathrm{pH}$ values of 3,5 , and 7 , respectively. These $D_{3 \mathrm{D}}$ values are the mean values of the diffusion coefficients of three different single particles calculated for the same experimental conditions. The calculated values of $D_{3 \mathrm{D}}$ increase significantly with increasing $\mathrm{pH}$, with the $D_{3 \mathrm{D}}$ at $\mathrm{pH} 7$ being roughly 70 times higher than that at $\mathrm{pH} 3$, reflecting the weaker attractive interaction forces between the particles and the glass surface at the higher $\mathrm{pH}$.

\section{Two types of 3D trajectories of single ceria particles, one with confined and one with Brownian motion, and a third type of an agglomerated cluster, all at pH 7}

Two entirely different particle motions of single ceria particles in $2 \mathrm{D}(x-y)$ plane were observed at $\mathrm{pH} 7$ [12]. As explained above, the motion of particle 1 was similar to the confined motion that was observed at $\mathrm{pH} 3$ and 5. In the second case (particle 2), the particle moved rapidly and moved far away from the glass surface and sometimes disappeared from the field of view. Therefore, the second particle could be tracked for a time period of only $5 \mathrm{~s}$ which yielded a sequence consisting of only 125 images that covered the space between $\sim-1500$ and $\sim 1500 \mathrm{~nm}$ in the $x$ - and $y$-directions and between $\sim 5$ and $\sim 270 \mathrm{~nm}$ in the $z$-direction [Fig. 5(a)]. In contrast, the particle showing confined motion could be tracked for the complete $20 \mathrm{~s}$ since it moved in a much smaller area of $300 \times 300 \mathrm{~nm}^{2}$ and smaller vertical distance along a $z$-axis of $150 \mathrm{~nm}$ (Fig. 3). Analysis of the $\mathrm{MSD}_{3 \mathrm{D}}$ [Fig. 5(c)] of the second particle showed that it is undergoing Brownian motion since the exponent $a$ is $\sim 1$, while the first particle is undergoing confined motion since $a$ is 0.14 as shown in Fig. 4. This difference is a consequence of the zeta-potential of ceria particles at $\mathrm{pH} 7$ being close to $0 \mathrm{mV}$ [12]. This means that while both positive and negative charges exist on the particle surface at the same time in different regions, they counterbalance each other. Several authors reported the non-uniform distribution of zetapotential on the particle surface and their standard deviation among different regions [29,30,31]. In our case, if it so happens that the attractive interaction of the positively charged parts on the surface of a specific particle with the negatively charged 


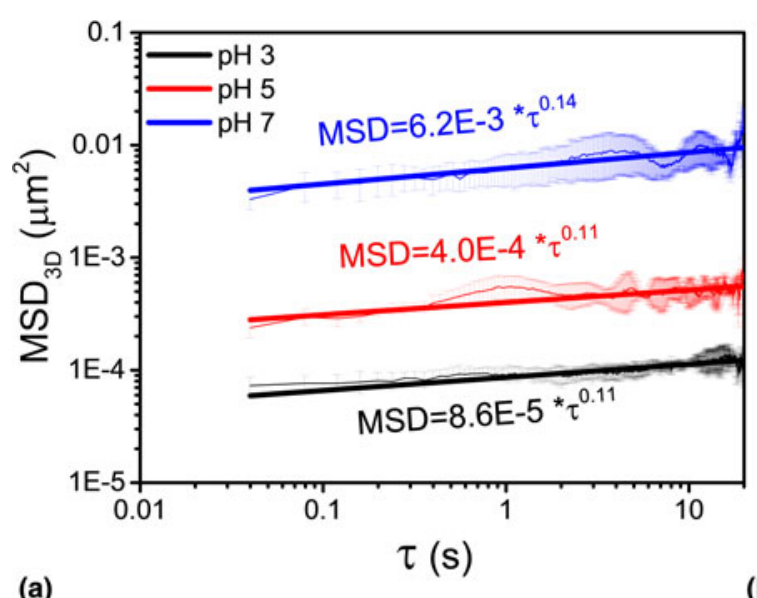

(a)

(b)
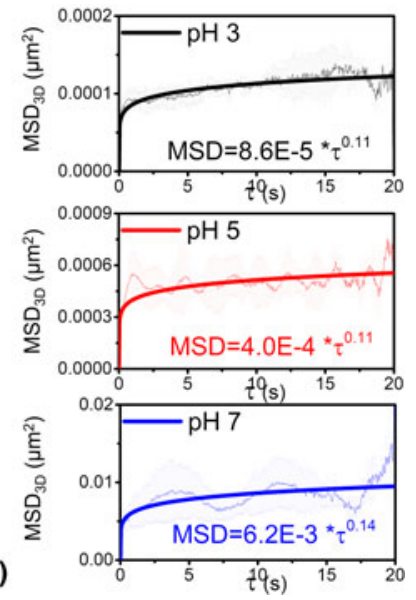

Figure 4: Mean square displacement, $M \mathrm{MD}_{3 \mathrm{D}}$ as a function of lag time $\tau$. (a) log-log plots and (b) linear-linear plots. The data were fitted to $M \mathrm{SD}_{3 \mathrm{D}}=6 \mathrm{D}_{3 \mathrm{D}} \mathrm{T}^{a}$, where $D_{3 \mathrm{D}}$ is the three-dimensional diffusion coefficient. The exponent $a$ was found to be $0.11,0.11$, and 0.14 for the three different $\mathrm{pH}$ values of 3,5 , and 7 , respectively. The shaded regions around each line indicate the ranges for MSD determined from the data on three different particles. (a)
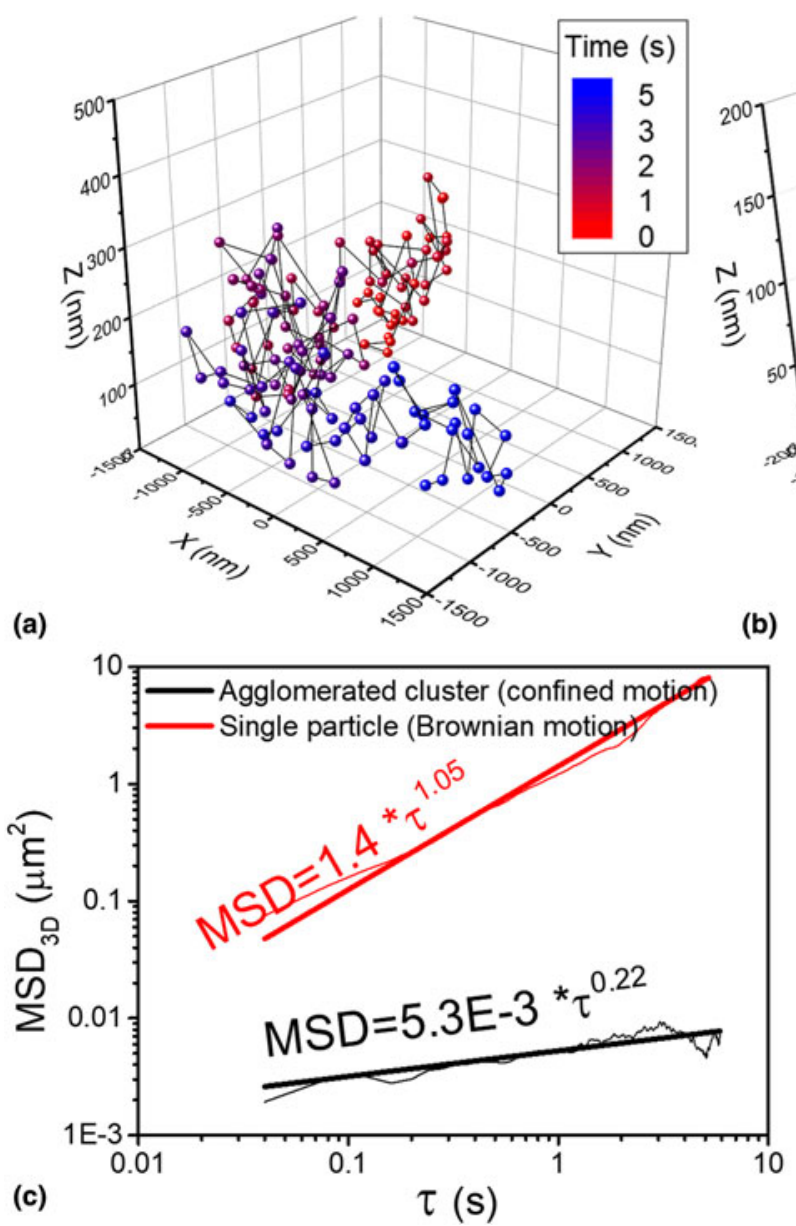

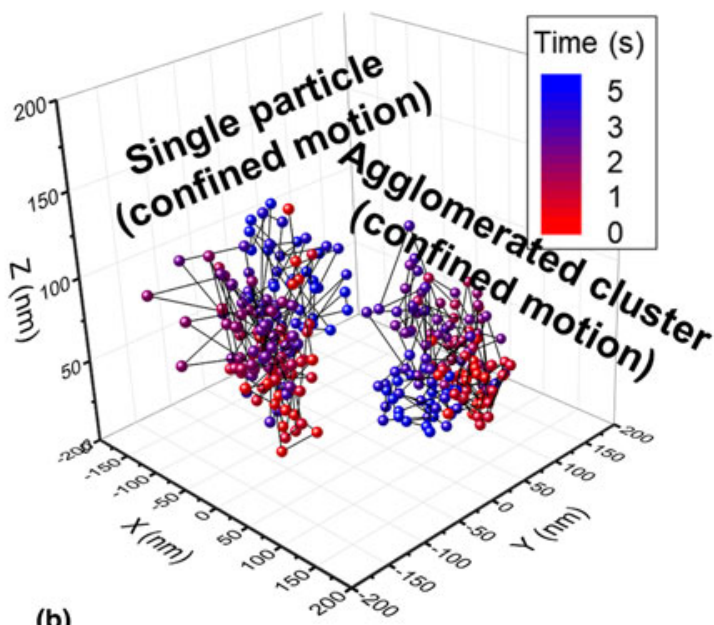

(b)
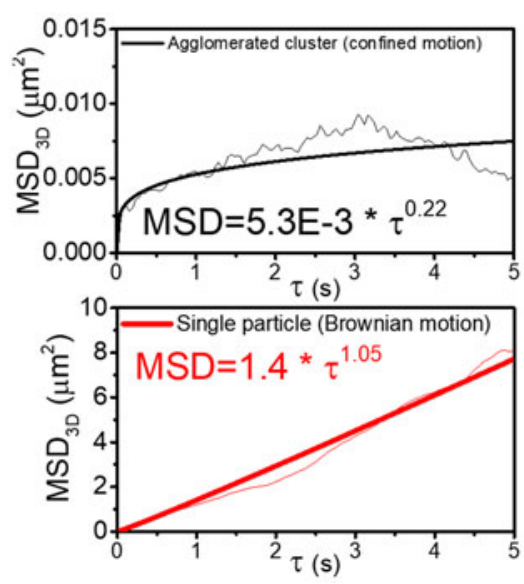

Figure 5: 3D trajectories of (a) single ceria particle with Brownian motion and (b) agglomerated cluster and single ceria particle with confined motion all at $\mathrm{pH} 7$ and (c) the corresponding $\mathrm{MSD}_{3 \mathrm{D}}$ data as a function of $\mathrm{\tau}$; log-log plots and linear-linear plots. The exponent $a$ for $\mathrm{MSD}_{3 \mathrm{D}}$ for single ceria particle with Brownian motion (red) and an agglomerated cluster (black) are 1.05 and 0.22, respectively. 3D trajectory of the single particle with confined motion is the same as that shown in Fig. 3.

glass surface is dominant, then it results in confined motion. If either the local repulsion induced by the negative charge on the particle surface or the weakened attraction due to the net value of the zeta-potential being close to zero is dominant, then the particle can move further away from the glass surface resulting in Brownian motion. In such a case, the weakened interaction 
TABLE 1: Summary of $D_{2 \mathrm{D}}$ and $D_{3 \mathrm{D}}$ of ceria particles near a glass surface at $\mathrm{pH}$ 3,5 , and 7 obtained from their respective MSD data as a function of lag time $\tau$. $D_{2 \mathrm{D}}$ values are from our previous paper [12].

\begin{tabular}{llll}
\hline \hline $\mathrm{pH}$ & Motion & $D_{2 \mathrm{D}}\left(\mu \mathrm{m}^{2} / \mathrm{s}\right)$ & $D_{3 \mathrm{D}}\left(\mu \mathrm{m}^{2} / \mathrm{s}\right)$ \\
\hline $\mathrm{pH} \mathrm{3}$ & Confined & $1.8 \times 10^{-5}$ & $1.4 \times 10^{-5}$ \\
$\mathrm{pH} 5$ & Confined & $8.3 \times 10^{-5}$ & $6.7 \times 10^{-5}$ \\
$\mathrm{pH} 7$ & Confined & $1.2 \times 10^{-3}$ & $1.0 \times 10^{-3}$ \\
& Brownian & $3.8 \times 10^{-2}$ & $2.0 \times 10^{-1}$ \\
$\mathrm{pH} \mathrm{7-agglomerated}$ & Confined & $1.1 \times 10^{-3}$ & $8.8 \times 10^{-4}$ \\
\hline \hline
\end{tabular}

allows the particle to move farther and faster from the glass surface as seen in our measurements. As such, the particle with Brownian motion can move $\sim 270 \mathrm{~nm}$ away from the glass surface, while the particle with confined motion can only move up to $\sim 150 \mathrm{~nm}$.

Additionally, since the zeta-potential of ceria particle at $\mathrm{pH}$ 7 is close to $0 \mathrm{mV}$, particle agglomeration occurs. Agglomeration of particles in colloidal dispersions is well known and occurs when the zeta-potential is very low and is caused by the dominance of van der Waals attraction [12,32, 33]. Among many agglomerated clusters observed in the imaged area, one such agglomerated cluster (particle 3) was chosen as a representative sample. The $2 \mathrm{D}$ trajectory of the agglomerated cluster (particle 3) was obtained by tracking local highest EW scattering intensity (closest to the glass surface) of the cluster and fitting it to a Gaussian function using the particle tracking software [17] and then combined with the distance of the agglomerated cluster from the glass surface in the $z$-direction to generate its 3D trajectory [Fig. 5(b)]. Analysis of the $\mathrm{MSD}_{3 \mathrm{D}}$ of this cluster showed that it is undergoing confined motion since $a$ is $\sim 0.2$ [Fig. 5(c)].

The $D_{3 \mathrm{D}}$ of the single particle undergoing Brownian motion $\left(2.0 \times 10^{-1} \mu \mathrm{m}^{2} / \mathrm{s}\right)$ is about 200 times higher than that of the particle with confined motion $\left(1.0 \times 10^{-3} \mu \mathrm{m}^{2} / \mathrm{s}\right)$. The $D_{3 \mathrm{D}}$ of the agglomerated cluster $\left(8.8 \times 10^{-4} \mu \mathrm{m}^{2} / \mathrm{s}\right)$ is only slightly lower than that of the particle with the confined motion, perhaps because of the gravitational attraction. The $D_{3 \mathrm{D}}$ values are summarized in Table 1 along with the $D_{2 \mathrm{D}}$ values that were calculated earlier from $\mathrm{MSD}_{2 \mathrm{D}}$ curves $(\mathrm{MSD}=$

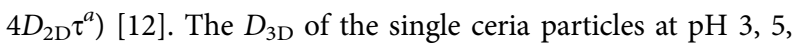
and 7 and of the agglomerated clusters at $\mathrm{pH} 7$ showing confined motion were slightly lower than their $D_{2 \mathrm{D}}$ (Table 1), presumably due to the attractive interaction forces along the $z$-directions.

The bulk diffusion coefficient $\left(D_{0}\right)$ of the ceria particles obtained from dynamic laser scattering (DLS) measurements based on the Stokes-Einstein equation has a value of $3.0 \mu \mathrm{m}^{2} / \mathrm{s}$ [12], but the $D_{3 \mathrm{D}}$ of even the particle exhibiting Brownian motion at $\mathrm{pH} 7$ calculated from $\mathrm{MSD}_{3 \mathrm{D}}$ is only $0.2 \mu \mathrm{m}^{2} / \mathrm{s}$ [Fig. 5(c)], a value that is 15 times smaller. The diffusion coefficients along $x$-, $y$-, and $z$-directions of a particle undergoing Brownian motion used in DLS experiments are the same, $D x=D y=D z$, due to the homogeneous environment. Also, the equation used to calculate diffusion coefficients using DLS does not consider any external forces (e.g., repulsive or attractive forces) acting on a particle. However, our system here is aimed at studying particle-surface interactions that influence particle motion, and the much lower $D_{3 \mathrm{D}}$ values obtained from our experiments as compared to $D_{0}$ are likely due to the electrostatic attractive forces prevailing between ceria particles and glass surface.

In the next section, we will discuss how the $\mathrm{pH}$-dependent 3D trajectories, three-dimensional diffusion coefficients, and interaction potential energy data (obtained in our previous paper [12]) of ceria particles near a glass surface are related to their removal by repeated washing with DI water adjusted to $\mathrm{pH} 10$ and 12 .

\section{Removal of ceria particles from a glass substrate as a function of the number of washes at $\mathrm{pH} 10$ and 12}

Ceria particle-based slurries have been widely used for $\mathrm{SiO}_{2}$ polishing, especially for shallow trench isolation (STI) chemical mechanical planarization (CMP) because of their high chemical affinity toward $\mathrm{SiO}_{2}$ films [34]. However, this affinity presents a major challenge for post-CMP cleaning as the particles strongly adhere to the wafer surface during polishing $[5,35]$. With device dimensions, now at $7 \mathrm{~nm}$, continuing to shrink, there is a strong need to understand various chemical and mechanical phenomena that occur in the brush cleaning systems used to remove these particles. Here, we show the relationship between 3D trajectories, three-dimensional diffusion coefficients, and interaction potential energy data of ceria particles and particle cleaning using $\mathrm{pH}$-adjusted de-ionized water (DIW), as imaged by EW spectroscopy. While the actual post-CMP cleaning of oxide surfaces is a very complex engineering process, the results obtained here provide valuable insights. As stated earlier, the flat glass surface of the lens became our model for the $\mathrm{SiO}_{2}$ film surface of interest here.

$50 \mu \mathrm{L}$ of a ceria slurry was placed on the glass surface and imaged after 20 min. Figure 6 displays the EW images of ceria particles left on the glass surface (covering an area of $77 \times$ $77 \mu \mathrm{m}^{2}$ ) at $\mathrm{pH} \mathrm{3,5}$, and 7 and cleaned for $40 \mathrm{~s}$ using a water jet from a simple spray bottle containing DIW adjusted to $\mathrm{pH} 10$ and 12 and then imaged as a function of the number of washes. The higher $\mathrm{pH}$ values were chosen for cleaning to benefit from the repulsive forces at these values between ceria particles and glass surfaces. The number of ceria particles initially present on the glass surface decreased with an increase in $\mathrm{pH}$ ( $\sim 2950$ at $\mathrm{pH} \mathrm{3,} \sim 530$ at $\mathrm{pH} \mathrm{5,} \mathrm{and} \sim 427$ at $\mathrm{pH} 7)$. After cleaning, the number of residual ceria particles on the glass 
(a)

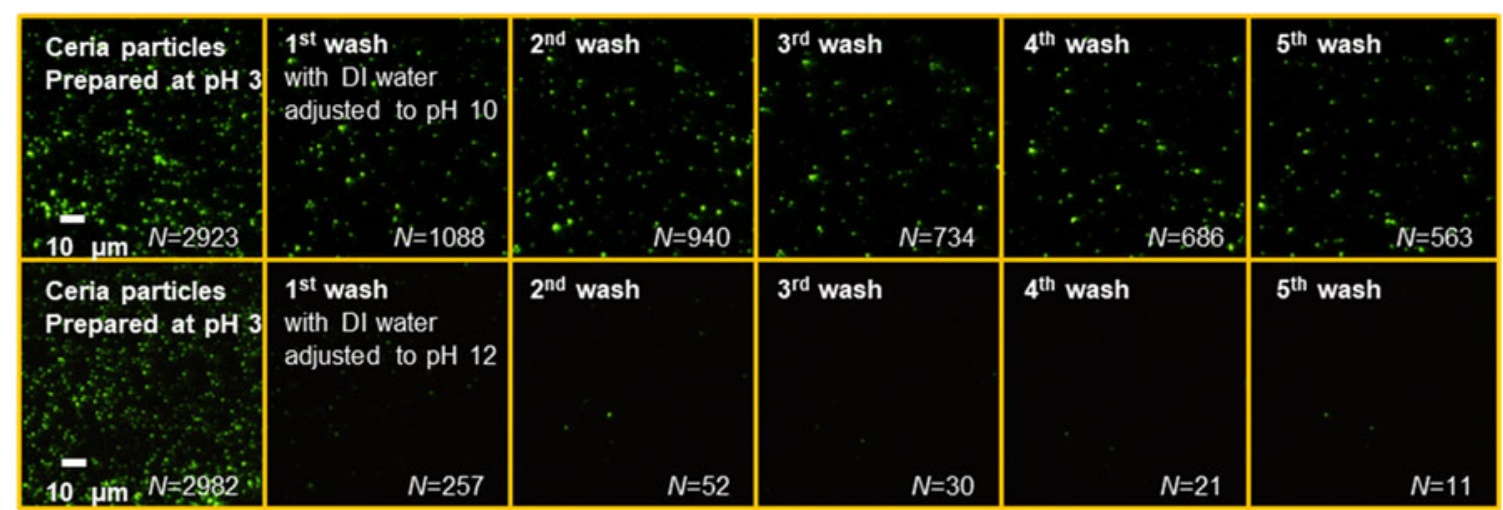

(b)
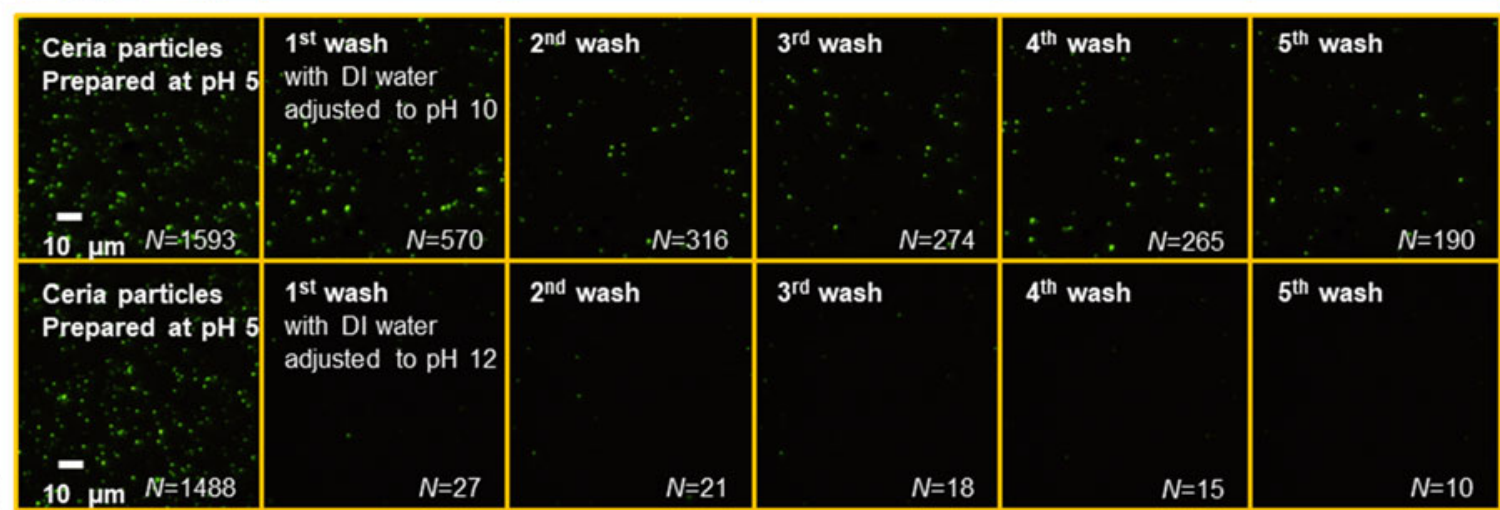

$5^{\text {th }}$ wash
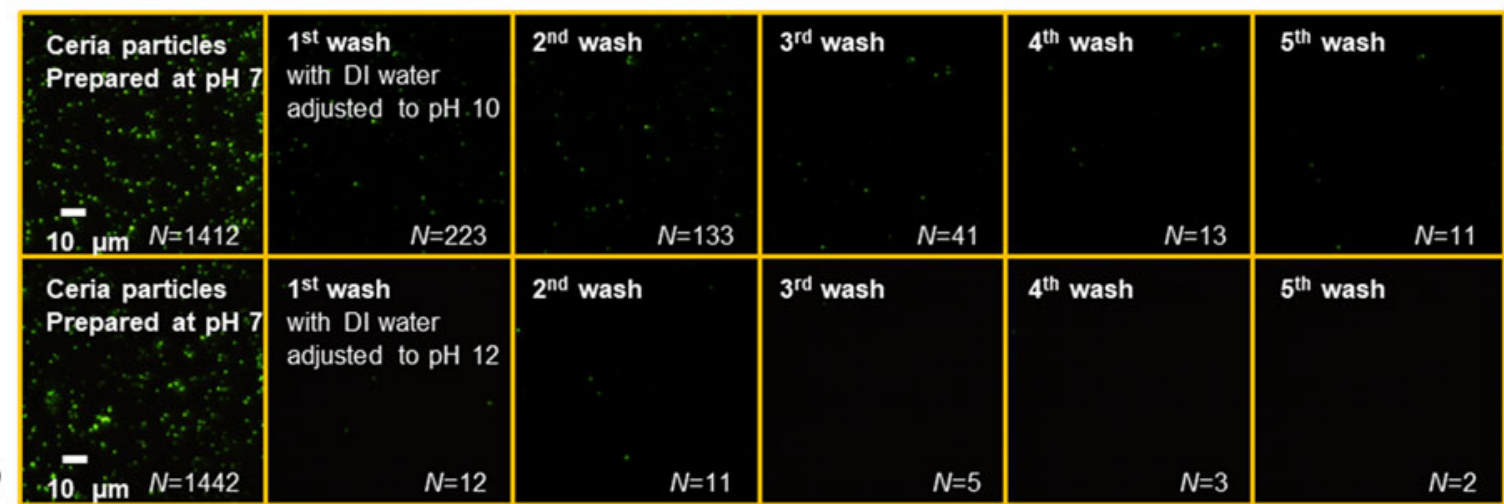

Figure 6: EW microscopy images of ceria particles adsorbed at (a) $\mathrm{pH} 3$, (b) $\mathrm{pH} 5$, (c) $\mathrm{pH} 7$ and their removal using DIW adjusted to $\mathrm{pH} 10$ and 12 as a function of number of washes. The first image in each row was captured 20 min after placing the sample on the lens. The subsequent images were obtained after washing the particles from the lens. The first and second rows in each panel display the EW scattering images of residual ceria particles after washing with DIW adjusted to pH 10 and 12 , respectively. $N$ is the number of ceria particles on the glass surface.

surface was determined as a function of the number of washes at both pH 10 and 12 (Fig. 7). The corresponding cleaning efficiencies, defined as the number of particles remaining as a percent of the particles initially present, are summarized in the inset in Fig. 7.

Cleaning efficiencies of DIW adjusted to $\mathrm{pH} 10$ and 12 for removal of ceria particles from the samples prepared at $\mathrm{pH} 3$ reached $88 \%$ and $99 \%$ [Fig. 6(a)], respectively, after 8 washes. In contrast, particles on the samples prepared at $\mathrm{pH} 5$ [Fig. 6 (b)] and pH 7 [Fig. 6(c)] can be cleaned with higher efficiencies at both $\mathrm{pH} 10$ and 12 after only 3 or 4 washes. These results are consistent with the above 3D trajectory data and the measured interaction potential energy data [12] that showed that ceria particles at $\mathrm{pH} 3$ can remain closer to the glass surface making them more difficult to be cleaned when compared to particles at $\mathrm{pH} 5$ and 7 which can move farther and faster from the glass surface due to the weakening of the attractive forces [12]. Earlier, we demonstrated the ceria particles adsorbed on oxide surfaces via strong $\mathrm{Ce}-\mathrm{O}-\mathrm{Si}$ bond could not be removed effectively by only charge repulsion [5]. $\mathrm{pH}$-adjusted DIW ( $\mathrm{pH}$ 13.5) showed negligible cleaning efficiency $(<5 \%)$ for $90 \mathrm{~nm}$ ceria particles, even when aided by ultrasonic cleaning [5]. In 


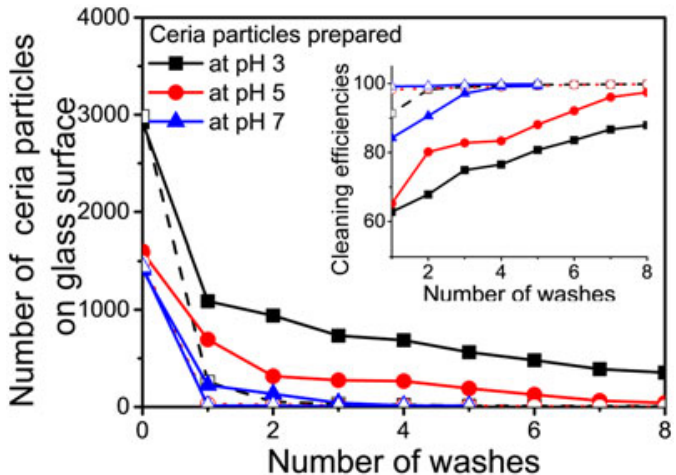

Figure 7: Number of ceria particles calculated from EW microscopy images shown in Fig. 6 at (a) pH 3, (b) pH 5, and (c) pH 7 and their removal as a function of the number of washes using DIW adjusted to $\mathrm{pH} 10$ (filled symbols) and pH 12 (open symbols). Inset shows the corresponding cleaning efficiencies.

this study, ceria particles move near the glass surface at $\mathrm{pH} 3,5$, and 7 without the formation of strong $\mathrm{Ce}-\mathrm{O}-\mathrm{Si}$ bonds with the glass surface, and hence DIW adjusted to $\mathrm{pH} 10$ and 12 was able to remove them from the surface by only charge repulsion. While charge repulsion is known to be a key driver of cleaning performance, our data provide a quantitative measure of this effect. This is especially true for the larger particles used here since we recently showed that smaller (sub-50 nm) ceria particles with a higher surface concentration of $\mathrm{Ce}^{3+}$ adsorbed on oxide surfaces cannot be removed effectively by only charge repulsion because they are more strongly coupled with oxide surfaces via strong Ce-O-Si bonds [5]. Such smaller particles can be removed only after breaking this bond and not by just relying on electrostatic repulsion [5].

\section{D versus 2D data analysis and results}

One can argue that the calculation of MSD of particles traveled in $3 \mathrm{D}$, and the $3 \mathrm{D}$ diffusion coefficients did not provide any new insights beyond those obtained from a $2 \mathrm{D}$ analysis. However, this in itself is remarkable since we would not have predicted it beforehand and may not apply to all systems. We find, for example, that while particles are easily removed during cleaning, significant particle redeposition occurs and prevents complete particle removal using a rotating PVA brush, a common practice in the industry to clean wafers post-CMP. Particle redeposition is determined by the diffusion and/or convective transport in the thin liquid film, where the $3 \mathrm{D}$ trajectories and diffusion coefficients matter, between the glass and the porous PVA brush surfaces. These processes are being imaged and analyzed now using EW spectroscopy [16].

Furthermore, using our extended methodology and based on the techniques developed to study brush cleaning, it should be possible to image pad/wafer and pad/abrasive (ceria or silica)/wafer contact during polishing-something that has not been possible so far-using model wafer coupons and polishing pad pieces. Finally, one might be able to investigate the behavior of smaller ceria and other particles using only 2D data since gravitational effects will be lower.

\section{Conclusions}

$\mathrm{pH}$-dependent 3D trajectories, diffusion coefficients, and interactions of ceria particles with a glass surface, representing a $\mathrm{SiO}_{2}$ surface, were investigated using EW microscopy with implications for ceria particle cleaning on $\mathrm{SiO}_{2}$ surfaces relevant to semiconductor manufacturing processes. Ceria particles showed only confined or sub-diffusive motion at $\mathrm{pH} 3$ and 5, while both confined and Brownian motion were exhibited at $\mathrm{pH}$ 7. The $D_{3 \mathrm{D}}$ of ceria particles showing confined motion increased with $\mathrm{pH}$ due to weaker attractive forces between ceria particles and the glass surface at higher $\mathrm{pH}$ values. The $D_{3 \mathrm{D}}$ of the particle undergoing Brownian motion $(2.0 \times$ $10^{-1} \mu \mathrm{m}^{2} / \mathrm{s}$ ) at $\mathrm{pH} 7$ was almost 200 times higher than that exhibiting confined motion $\left(1.0 \times 10^{-3} \mu \mathrm{m}^{2} / \mathrm{s}\right)$ at the same $\mathrm{pH}$. The $D_{3 \mathrm{D}}$ values of agglomerated clusters and single ceria particles exhibiting confined motion were slightly lower than the $D_{2 \mathrm{D}}$ values because of the attractive forces along the $z$-directions. The $D_{3 \mathrm{D}}$ values from experiments were much lower than the bulk diffusion coefficient, likely due to the attractive forces between ceria particles and the glass surface. Ceria particles on the glass surfaces deposited at high $\mathrm{pH}$ were more easily removed from glass surfaces during the cleaning process due to the weaker attractive interaction forces between the particles and the glass surface consistent with their $3 \mathrm{D}$ trajectories.

\section{Experimental Techniques, Materials, and Experimental Procedures}

\section{EW microscopy setup and data analysis}

In our experimental setup, an EW is generated when the laser light beam having $15 \mathrm{~mW}$ power at $532 \mathrm{~nm}$ wavelength (Edmund Optics, Barrington, NJ, USA) undergoes total internal reflection at the glass lens-ceria suspension interface [12, $13,14]$. The incident angle $\theta_{\mathrm{i}}$ was adjusted to $69.5 \pm 1.0^{\circ}$, and the penetration depth $\beta$ of the EW field was $88.0 \pm 4.5 \mathrm{~nm}$. The penetration depth was calculated as $\beta=\lambda / 4 \pi\left(n_{1}^{2} \sin ^{2} \theta_{i}-n_{2}\right.$ 2) $-1 / 2$, where $\lambda$ is the wavelength of the incident light, $\theta_{i}$ is the incident angle, and $n_{1}$ and $n_{2}$ are the refractive indices of glass and suspension, respectively. The EW scattered light passes through a $50 \times$ objective lens (Nikon, Japan) and hits a dielectric coated right-angled prism mirror where it gets reflected. The reflected light is refocused by an infinity-corrected tube lens (Thorlabs, USA). The particles in the sample suspension placed on the glass lens surface are detected using a CMOS 
camera (Baumer, USA) at a resolution of 13.37 pixels $/ \mu \mathrm{m}$. This homemade EW microscopy system was used to image interactions of ceria particles near a glass lens surface in real time using the software microDisplay 5.2.2 (Silicon Software $\mathrm{GmbH}$, Germany). The videos captured 500 images with dimensions of $1024 \times 1024$ pixels $\left(77 \times 77 \mu^{2}\right)$ for $20 \mathrm{~s}$. The EW images and videos were analyzed using the particle tracking software Diatrack v3.04 (Semasopht, Switzerland) [36]. Particles were detected by fitting 2D Gaussian functions based on a specified intensity threshold. 2D trajectories of a single-specific ceria particle were obtained by linking particle's positions (i.e., centroids) as a function of time. The closest distance $h$ between a particle and the glass surface can be determined using the relation between intensity and height: $I(h)=$ $I_{0} \exp \left(-\beta^{-1} h\right)$ [18], where $I_{0}$ is the intensity at the ceria particle-glass surface contact and $\beta$ is the penetration depth (88.0 $\pm 4.5 \mathrm{~nm}$ ). The $3 \mathrm{D}$ positions of these particles were obtained by combining the separation distance $h$ in the $z$-direction with the positions in the $2 \mathrm{D}(x-y)$ plane. $3 \mathrm{D}$ trajectories of these particles were then constructed by linking their timesequenced positions. Three-dimensional mean square displacement $\left(\mathrm{MSD}_{3 \mathrm{D}}\right)$ as a function of $\tau$ was calculated and plotted using Labview (National Instrument Corp, USA). All experiments were performed at room temperature to minimize laserinduced heating of the particles. The heating of the particles due to the absorption of the laser light can be affected by the wavelength and power of the light source and the environmental conditions. Given the results reported by Liu et al. [37,38], less than $1^{\circ} \mathrm{C}$ change in the local temperature at the particleglass interface in our experiments conditions is expected, which is negligible. The details of EW microscopy setup, the experimental conditions, and data analysis were reported in our earlier publications $[12,13,14]$.

\section{Materials and experimental procedures}

Ceria slurry with a mean particle diameter of $\sim 140 \mathrm{~nm}$ was supplied by Ferro Electronic Materials. A dilute suspension of ceria particles $(0.005 \mathrm{wt} \%)$ was prepared and subsequently sonicated to minimize particle aggregation. $\mathrm{pH}$ of the ceria suspension and DIW was adjusted to the desired value using $\mathrm{HNO}_{3}$ or $\mathrm{KOH} .50 \mu \mathrm{L}$ of the ceria suspension was placed on the glass lens surface and imaged in real time using EW microscopy. First, a background or baseline image of the lens (without any liquid) was obtained. $50 \mu \mathrm{L}$ of ceria slurry was then placed on the glass surface and imaged after waiting for 20 min to determine the number of the ceria particles on the glass surface.

The lens covered with ceria particles was removed from the lens mount and subsequently washed multiple times, each time with $\sim 40 \mathrm{~mL}$ of a cleaning solution (DIW adjusted to $\mathrm{pH} 10$ and $\mathrm{pH} 12$ ) sprayed from a squeeze bottle for $4 \mathrm{~s}$ (flow rate of $\sim 10 \mathrm{~mL} / \mathrm{s}$ ) and then imaged. The number of particles on the lens surface was calculated before and after each wash from the EW images using Diatrack v3.04 and the particle cleaning efficiencies were determined from these values.

\section{Acknowledgments}

The authors gratefully acknowledge EBARA Corporation for providing partial financial support and Ferro for supplying ceria slurry.

\section{References}

1. K.L. Mittal: Particles on Surfaces: Detection, Adhesion and Removal, Vol. 9 (CRC Press, Boca Raton, FL, 2006).

2. A.M. Freitas and M.M. Sharma: Detachment of particles from surfaces: An AFM study. J. Colloid Interface Sci. 233, 73 (2001).

3. X. Gong, Z. Wang, and T. Ngai: Direct measurements of particlesurface interactions in aqueous solutions with total internal reflection microscopy. Chem. Commun. 50, 6556 (2014).

4. M. Ranade: Adhesion and removal of fine particles on surfaces. Aerosol Sci. Technol. 7, 161 (1987).

5. J. Seo, A. Gowda, and S. Babu: Almost complete removal of ceria particles down to $10 \mathrm{~nm}$ size from silicon dioxide surfaces. ECS J. Solid State Sci. Technol. 7, P243 (2018).

6. J. Seo, J.W. Lee, J. Moon, W. Sigmund, and U. Paik: Role of the surface chemistry of ceria surfaces on silicate adsorption. ACS Appl. Mater. Interfaces 6, 7388 (2014).

7. J.T. Abiade, W. Choi, and R.K. Singh: Effect of $\mathrm{pH}$ on ceria-silica interactions during chemical mechanical polishing. J. Mater. Res. 20, 1139 (2005).

8. S. Korkmaz, A.S. Vahdat, O. Trotsenko, S. Minko, and S. Babu: AFM-based study of the interaction forces between ceria, silicon dioxide and polyurethane pad during non-prestonian polishing of silicon dioxide films. ECS J. Solid State Sci. Technol. 4, P5016 (2015).

9. Q.K. Ong and I. Sokolov: Attachment of nanoparticles to the AFM tips for direct measurements of interaction between a single nanoparticle and surfaces. J. Colloid Interface Sci. 310, 385 (2007).

10. E.R. Borujeny, M. Miao, H. Pirayesh, Z. Xu, and K. Cadien: An investigation of the deposition of ceria on silica by quartz crystal microbalance: Observations on the effect of many body interactions. Colloids Surf. A 522, 207 (2017).

11. X. Liu, G. Chen, and C. Su: Influence of collector surface composition and water chemistry on the deposition of cerium dioxide nanoparticles: QCM-D and column experiment approaches. Environ. Sci. Technol. 46, 6681 (2012).

12. J. Seo, A. Gowda, P. Khajornrungruang, S. Hamada, T. Song, and S. Babu: Trajectories, diffusion, and interactions of single 
ceria particles on a glass surface observed by evanescent wave microscopy. J. Mater. Res., 35, 321 (2020).

13. A. Pal, P. Khajornrungruang, C. Netzband, S. Alety, and S. Babu: Observation of the formation of anisotropic silver microstructures by evanescent wave and electron microscopy. Nanotechnology 27, 075708 (2016).

14. P. Khajornrungruang, S. Korkmaz, P. Angshuman, K. Suzuki, K. Kimura, and S.V. Babu: Light scattering model for individual sub-100-nm particle size determination in an evanescent field. Jpn. J. Appl. Phys. 55, 06JG02 (2016).

15. M. Hanai, T. Sanada, A. Fukunaga, and H. Hiyama: Observation of the real contact area between PVA brush and surface using polarization plate and evanescent field. In ICPT 2017: International Conference on Planarization/CMP Technology (VDE, Frankfurt, Germany, 2017), p. 1.

16. Y. Terayama, P. Khajornrungruang, K. Suzuki, K. Kusatsu, S. Hamada, Y. Wada, and H. Hiyama: Real time nanoscale cleaning phenomenon observation during PVA brush scrubbing by evanescent field. ECS Trans. 92, 191 (2019).

17. P. Vallotton, A.M. Van Oijen, C.B. Whitchurch, V. Gelfand, L. Yeo, G. Tsiavaliaris, S. Heinrich, E. Dultz, K. Weis, and D. Grünwald: Diatrack particle tracking software: Review of applications and performance evaluation. Traffic 18, 840 (2017).

18. D.C. Prieve: Measurement of colloidal forces with TIRM. Adv. Colloid Interface Sci. 82, 93 (1999).

19. D.C. Prieve and J.Y. Walz: Scattering of an evanescent surface wave by a microscopic dielectric sphere. Appl. Opt. 32, 1629 (1993).

20. L. Liu, A. Woolf, A.W. Rodriguez, and F. Capasso: Absolute position total internal reflection microscopy with an optical tweezer. Proc. Natl. Acad. Sci. 111, E5609 (2014).

21. C.T. McKee, S.C. Clark, J.Y. Walz, and W.A. Ducker: Relationship between scattered intensity and separation for particles in an evanescent field. Langmuir 21, 5783 (2005).

22. C. Hertlein, N. Riefler, E. Eremina, T. Wriedt, Y. Eremin,

L. Helden, and C. Bechinger: Experimental verification of an exact evanescent light scattering model for TIRM. Langmuir 24, 1 (2008).

23. C. Gell, M. Berndt, J. Enderlein, and S. Diez: TIRF microscopy evanescent field calibration using tilted fluorescent microtubules. J. Microsc. 234, 38 (2009).

24. F. Huang, E. Watson, C. Dempsey, and J. Suh: Real-time particle tracking for studying intracellular trafficking of pharmaceutical nanocarriers. In Cellular and Subcellular Nanotechnology, V. Weissig, T. Elbayoumi and M. Olsen, eds. (Springer, Berlin, Germany, 2013); pp. 211.
25. J-H. Jeon, N. Leijnse, L.B. Oddershede, and R. Metzler: Anomalous diffusion and power-law relaxation of the time averaged mean squared displacement in worm-like micellar solutions. New J. Phys. 15, 045011 (2013).

26. D. Ben-Avraham and S. Havlin: Diffusion and Reactions in Fractals and Disordered Systems (Cambridge University Press, Cambridge, UK, 2000).

27. S. Havlin and D. Ben-Avraham: Diffusion in disordered media. Adv. Phys. 36, 695 (1987).

28. F. Höfling and T. Franosch: Anomalous transport in the crowded world of biological cells. Rep. Prog. Phys. 76, 046602 (2013).

29. D. Velegol, J.D. Feick, and L.R. Collins: Electrophoresis of spherical particles with a random distribution of zeta potential or surface charge. J. Colloid Interface Sci. 230, 114 (2000).

30. J.D. Feick and D. Velegol: Electrophoresis of spheroidal particles having a random distribution of zeta potential. Langmuir 16, 10315 (2000).

31. D. Velegol and P.K. Thwar: Analytical model for the effect of surface charge nonuniformity on colloidal interactions. Langmuir 17, 7687 (2001).

32. J. Seo and U. Paik: Preparation and characterization of slurry for chemical mechanical planarization (CMP) .In Advances in Chemical Mechanical Planarization (CMP), S.V. Babu, ed. (Elsevier, Amsterdam, The Netherlands, 2016); pp. 273.

33. W.M. Sigmund, N.S. Bell, and L. Bergström: Novel powderprocessing methods for advanced ceramics. J. Am. Ceram. Soc. 83, 1557 (2000).

34. R. Srinivasan, P.V. Dandu, and S. Babu: Shallow trench isolation chemical mechanical planarization: A review. ECS J. Solid State Sci. Technol. 4, P5029 (2015).

35. A. Gowda, J. Seo, C.K. Ranaweera, and S.V. Babu: Cleaning solutions for removal of $\sim 30 \mathrm{~nm}$ ceria particles from proline and citric acid containing slurries deposited on silicon dioxide and silicon nitride surfaces. ECSJ. Solid State Sci. Technol. 9, 044013 (2020).

36. P. Vallotton and S. Olivier: Tri-track: Free software for large-scale particle tracking. Microsc. Microanal. 19, 451 (2013).

37. Y. Liu, D. Cheng, G. Sonek, M. Berns, C. Chapman, and B. Tromberg: Evidence for localized cell heating induced by infrared optical tweezers. Biophys. J. 68, 2137 (1995).

38. Y. Liu, G. Sonek, M. Berns, and B. Tromberg: Physiological monitoring of optically trapped cells: assessing the effects of confinement by 1064-nm laser tweezers using microfluorometry. Biophys. J. 71, 2158 (1996). 